\title{
The Clinical Applications of Serum and Urinary Biomarkers in Prostate Cancer
}

\author{
Renu S. Eapen, ${ }^{\otimes 1}$ Peter E. Lonergan, ${ }^{2}$ Dominic Bagguley, ${ }^{3}$ Sean Ong, ${ }^{3}$ Ben Condon, ${ }^{3}$ \\ Nathan Lawrentschuk, 1,3,4,5 Maxwell V. Meng ${ }^{2}$ \\ ${ }^{1}$ Department of Genitourinary Oncology, Peter MacCallum Cancer Centre, Melbourne, Australia, ${ }^{2}$ Department of Urology, Helen Diller Family Comprehensive Cancer \\ Center, University of California, San Francisco, United States, ${ }^{3}$ EJ Whitten Prostate Cancer Research Centre at Epworth, Melbourne, Australia, ${ }^{4}$ Department of Surgery, \\ University of Melbourne, Australia, ${ }^{5}$ Department of Urology, Royal Melbourne Hospital, Australia
}

\begin{abstract}
At every stage of the prostate cancer journey from screening and diagnosis to management of advanced disease, patients and clinicians face dilemmas and decisions that can impact long-term outcomes. Although traditional risk stratification in prostate cancer is based on serum prostate specific antigen, clinical stage and Gleason score, in recent years, biomarkers have been developed that may be useful in several clinical scenarios. Biomarkers that can accurately predict an individual patient's risk, prognosis, and response to specific treatments could lead to improvements in decision-making and clinical care. Although there is evidence to support the use of biomarkers to guide management decisions, the optimal scenario in which to use them, how to interpret the results, and how to incorporate those results into clinical decision-making can be confusing. Nevertheless, in the era of personalized and precision medicine, it is important for clinicians to be aware of what tests are available, what clinical questions they seek to answer, and what limitations they have. This review focuses on the serum and urine biomarkers for the management of prostate cancer that have been under intense investigation in recent years.
\end{abstract}

\section{Introduction}

Prostate cancer $(\mathrm{PCa})$ is a heterogenous disease with a highly variable clinical course and behavior. Traditional risk stratification of PCa has been based on standard clinical parameters such as prostate specific antigen, clinical stage, and biopsy Gleason scores. Following the widespread use of PSA testing, the pendulum swung towards overdiagnosis of clinically insignificant PCa and the subsequent overtreatment with its associated morbidity [1]. Traditional models and nomograms, although allowing risk stratification of patients to a certain degree, do not allow accurate prediction of outcomes for an individual patient with PCa, leading to potential undertreatment of high-risk disease $[2,3]$. Because of these limitations, leading to discordant care, many areas of uro-oncology have recognized the need for the development of reliable biomarkers to aid decision-making in various challenging clinical contexts. Finding the "ideal" biomarker that can accurately predict a patient's individual risk and improve on existing, validated risk stratification tools has become an area of intense research interest in the last decade.

Biomarkers can be classified according to the source (eg, serum, urine, or tissue) (Table 1) or the clinical decision juncture at which they are used (Figure 1). In this 2-part summary, we review commercially available blood, urine and tissue-based biomarkers and summarize the currently available data on their use in a variety of clinical scenarios.

The first section will focus on the serum and urinary PCa biomarkers. Blood and urine sources have the advantage of being non-invasive, easily attainable, and convenient for patients and physicians. The subsequent article will focus on tissue-based biomarkers [4].

Key Words

Biomarkers, non-invasive, serum, urine, prediction

\section{Competing Interests}

None declared

\section{Article Information}

Received on July 1, 2020

Accepted on August 16, 2020

Soc Int Urol J. 2020;1(1):30-38 


$\begin{array}{ll}\text { Abbreviations } \\ \text { \%fPSA } & \text { percent free PSA } \\ \text { BPH } & \text { benign prostatic hyperplasia } \\ \text { csPCa } & \text { clinically significant prostate cancer } \\ \text { DRE } & \text { digital rectal examination } \\ \text { EPI } & \text { ExoDx Prostate Intelliscore } \\ \text { HOXC6 } & \text { homeobox C6 } \\ \text { PAP } & \text { prostatic acid phosphatase } \\ \text { PCa } & \text { prostate cancer } \\ \text { PSAD } & \text { PSA density } \\ \text { PSADT } & \text { PSA doubling time } \\ \text { PSAV } & \text { PSA velocity } \\ \text { SOC } & \text { standard of care } \\ \text { tPSA } & \text { total PSA }\end{array}$

\section{Serum Biomarkers}

\section{Prostate Specific Antigen}

Measurement of serum PSA is the most widely used test to aid in the detection of early prostate cancer, despite its well-known limitations, including high false-positive rates, poor specificity for prostate cancer, which lead to over diagnosis.

PSA is not tumor specific as it is secreted by both benign and malignant prostatic tissue. Therefore, apart from $\mathrm{PCa}$, many benign processes such as inflammation, benign prostatic hyperplasia (BPH), and trauma may lead to increased PSA values. PSA gained FDA approval for PCa screening in 1994 after it was shown to have higher sensitivity than prostatic acid phosphatase (PAP) in the detection of prostate cancer [5].

Historically, a serum PSA level above $4 \mathrm{ng} / \mathrm{mL}$ was the accepted cutoff to predict the potential presence of prostate cancer [6]. However, it has since been recognized that $20 \%$ of patients diagnosed with prostate cancer have a PSA lower than $4 \mathrm{ng} / \mathrm{mL}[7,8]$. Furthermore, it has been reported that when using a PSA threshold of $4 \mathrm{ng} / \mathrm{mL}$, the specificity in detecting PCa is only $12.8 \%$, leading to high false-positive rates and unnecessary biopsies [9]. To enable better discrimination between $\mathrm{PCa}$ and $\mathrm{BPH}$, the measurement of the ratio of free to total PSA was proposed as a more accurate indicator, and many studies have demonstrated its usefulness [10-13]. In a large prospective multicenter clinical trial of 773 patients with PSA levels between 2 and $10 \mathrm{ng} / \mathrm{mL}$, it was shown that a percent free PSA (\%fPSA) cutoff of 25\% detected 95\% of cancers and avoided $20 \%$ of unnecessary biopsies, with minimal loss in sensitivity [14]. A multivariable analysis showed \%fPSA to be an independent predictor of prostate cancer (OR 3.2, 95\% CI 2.5 to $4.1 ; P<0.001$ ) and more significant than total PSA (tPSA). Generally, the lower the \%fPSA, the higher the risk of cancer. However, prostate volume has been found to have an influence on \%fPSA. Stephan et al. reported diagnostic value of $\%$ fPSA in patients with prostate volume $<40 \mathrm{~cm}^{3}$, but advised caution in using \%fPSA in those with larger glands [15].

Other PSA metrics have also been employed to enhance the diagnostic and predictive capacity of PSA. These include PSA density (PSAD), PSA doubling time (PSADT), and PSA velocity (PSAV). PSA density is a quotient of serum PSA and prostate volume and may be a means of distinguishing between $\mathrm{BPH}$ and $\mathrm{PCa}$ [16]. A higher PSAD may not only indicate the presence of PCa but may also reflect the aggressiveness of the cancer. Studies have shown a correlation between higher PSAD and adverse PCa prognostic features [17]. A high PSAV and a shorter PSADT, especially in the setting of post-treatment biochemical recurrence (BCR), are associated with an increased risk of castration resistance, metastases, and cancer-specific and overall mortality [18].

\section{Prostate Health Index (PHI)}

Prostate Health Index (Beckman Coulter Inc., Brea, US) combines 3 quantitative kallikrein immunoassaystPSA, \%fPSA, and [-2]proPSA (p2PSA)-via a mathematical algorithm into a single score. The PHI received FDA approval in 2012 and is indicated as an adjunct to tPSA in men aged over 50 years with tPSA between 4 and $10 \mathrm{ng} / \mathrm{mL}$ and non-suspicious digital rectal examination (DRE) findings. Numerous validation studies have addressed the clinical utility of PHI. A prospective multi-institutional trial enrolled 892 men with no history of PCa, normal DRE, and tPSA of 2 to $19 \mathrm{ng} / \mathrm{mL}$, and found that within this range of PSA, at a sensitivity of $80 \%$ to $95 \%$, the specificity and area under the curve (AUC) of PHI exceeded those of PSA and \%fPSA [19]. Similarly, Stephan et al. evaluated 1362 patients with tPSA of 1.6 to $8.0 \mathrm{ng} / \mathrm{mL}$ who underwent systematic biopsy with $\geq 10$ cores, and showed that PHI $(\mathrm{AUC}=0.74)$ outperformed \%p2PSA $(\mathrm{AUC}=0.72)$, p2PSA $($ AUC $=0.63)$, \% fPSA $($ AUC $=0.61)$, and tPSA $(\mathrm{AUC}=0.56)$ in predicting $\mathrm{PCa}[20]$. Furthermore, these large series have demonstrated the advantage of PHI over \%fPSA in distinguishing between high-grade disease (Gleason score $\geq 4+3$ ) and low-grade disease or negative biopsies $[19,20]$. Catalona et al. showed that an increasing PHI was associated with a 4.7-fold increased risk of PCa and 1.6-fold increase in higher-grade disease. Other prospective studies have indicated the ability of PHI to detect aggressive (Gleason score $\geq 7$ ) cancer with higher specificity than tPSA and \%fPSA in biopsy naïve men, reducing the need for unnecessary biopsies [21].

The utility of $\mathrm{PHI}$ in detecting clinically significant prostate cancer (csPCa) was evaluated by Tosoian and colleagues, who reported that the median PHI density 


\section{TABLE 1.}

Commercially available biomarkers that are FDA and Clinical Laboratory Improvements Amendments (CLIA) approved
Biomarker
Molecular markers tested

Serum

Prostate Specific Antigen (PSA)*

Prostate Health Index (PHI)*

Beckman Coulter Inc, United States

4K score

OPKO Lab, United States
PSA

tPSA, \%fPSA, p2PSA

tPSA, fPSA, intact PSA, hK2

\section{Urine}

Progensa Prostate Cancer Antigen 3 (PCA3) ${ }^{*}$

Hologic, United States

PCA3

ExoDX Prostate (Intelliscore)

Exosome Diagnostics Inc, United States

PCA3, ERG

Michigan Prostate Score (MiPS), United States

TMPRSS2-ERG mRNA, PCA3

Select MDX

MDx Health, United States

HOXC6, DLX1

\section{Tissue}

\section{Confirm MDx}

MDxHealth, United States

\section{Oncotype Dx}

Genomic Health, United States

\section{Promark}

Metamark Genetics, United States

Prolaris*

Myriad Genetics, United States

Decipher

Genome Dx Biosciences, United States

* FDA-approved biomarkers

(based on prostate volume) was higher for those with csPCa (1.21) than for those with clinically insignificant $\mathrm{PCa}(0.70)$ and negative biopsies (0.53) with $P<0.001$. Using a threshold of 0.43 , PHI density was $97.9 \%$ sensitive and $38 \%$ specific for csPCa and $100 \%$ sensitive for Gleason $\geq 7$ disease [22]. The authors concluded that discriminative ability of PHI density $($ AUC $=0.84)$ for csPCa was higher than PHI, PSA, PSAD, \%fPSA, and prostate volume (AUC 0.52 to 0.79 ). Up to $38 \%$ of biopsies could be avoided while missing only $2 \%$ of
DNA methylation (GSTP1, APC, RASSF1, ACTB)

RNA (17 gene expression)

8 Proteins

RNA (46 gene expression)

RNA (22 gene expression)
csPCa [22]. Another advantage of PHI over \%fPSA is the lack of influence of patient age and prostate volume [19].

A recent study has evaluated the impact of $\mathrm{PHI}$ on clinical decision-making. In an observational study of 500 men, White et al. found that those who received a PHI test had a significantly lower biopsy rate compared to the control group $(36.4 \%$ versus $60.3 \% ; P<0.001)$. The PHI test purportedly impacted physicians' management plans, including the decision to defer biopsies when 


\section{FIGURE 1.}

Serum and urinary biomarkers according to their clinical applications

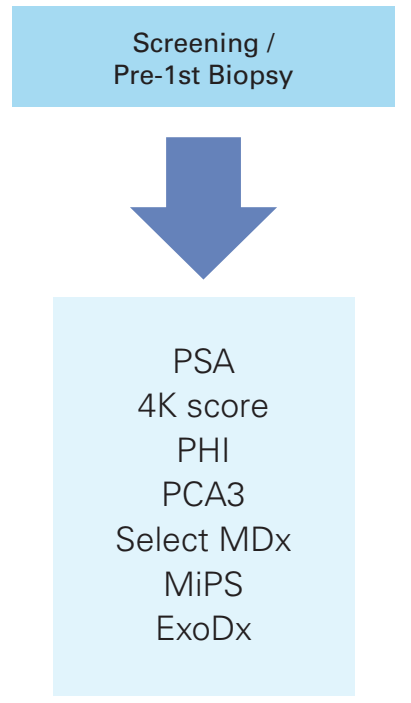

Previous Negative Biopsy / Pre-2nd Biopsy

PHI score was low and to perform biopsies when the PHI score was intermediate or high [23]. Similarly, the prospective comparative analysis of 345 men by Tosoian et al. demonstrated that PHI testing reduced the rate of biopsy procedures whilst the detection of higher-grade cancers remained unchanged [24].

Another potential application of the PHI score is the integration into a multivariable model with multi-parametric magnetic resonance imaging (mpMRI). Gnanapragasam et al. found that adding PHI to mpMRI improved the prediction of overall and csPCa (AUC 0.71 and 0.75) compared with mpMRI or PSA alone (AUC 0.64 and 0.69) [24]. In determining whether to re-biopsy men with a negative mpMRI, PHI performed better than PSA and PSAD in identifying csPCa. With a PHI threshold of $>35,42 \%$ of men avoided biopsy while missing only one of 21 significant cancers [25].

\section{$4 \mathrm{~K}$ Score}

The $4 \mathrm{~K}$ score (OPKO Health, Miami, US) comprises a panel of 4 kallikrein (4K) markers including tPSA, \%fPSA, intact PSA, and human kallikrein 2 (hK2) and combines clinical variables (age, DRE findings, and previous biopsy status) into a model to predict the likelihood of having csPCa (Gleason score $\geq 7$ ) on prostate biopsy. It may have value in reducing unnecessary prostate biopsies [25]. The merit of the $4 \mathrm{~K}$ score in the pre-biopsy setting has been extensively evaluated, particularly in Europe and the US [26-34].
Vickers et al. reported on 2914 men in the European Randomized Study of Screening for Prostate Cancer (ERSPC) who underwent prostate biopsy for PSA $\geq 3 \mathrm{ng} / \mathrm{mL}$, in which PCa was diagnosed in $28 \%$ of men [30]. Incorporating the $4 \mathrm{~K}$ score with PSA and age significantly improved predictive accuracy with (AUC 0.78 versus 0.70 ) and without (AUC 0.76 versus $0.64)$ DRE results $(P<0.001)$. For every 1000 men, the addition of the $4 \mathrm{~K}$ score would reduce the number of unnecessary biopsies by 513, albeit with the tradeoff that $12 \%$ of csPCa would be missed [30]. A smaller series of 262 men showed similar results, with the $4 \mathrm{~K}$ score having greater diagnostic accuracy than "base" clinical models incorporating PSA, age, and DRE findings only, with AUC increases from 0.63 to 0.78 in PCa prediction and 0.77 to 0.87 for prediction of high-grade $\mathrm{PCa}$ [27]. Parekh et al. prospectively evaluated the diagnostic performance of the $4 \mathrm{~K}$ score in 1012 men undergoing biopsy without restrictions on the indication for biopsy. Clinically significant $\mathrm{PCa}$ (Gleason $\geq 7$ ) was found in $23 \%$ of patients in this cohort [31]. The $4 \mathrm{~K}$ score outperformed the PCPT Risk Calculator 2.0 (AUC 0.82 versus $0.74 ; P<0.001$ ) in detecting csPCa and would have reduced the number of unnecessary biopsies by $58 \%$. In a large representative cohort study of 40379 men from Sweden, Stattin et al. studied the $4 \mathrm{~K}$ score for its ability to predict the risk of distant metastases [32]. Using a risk stratification approach to prostate biopsy using PSA and $4 \mathrm{~K}$ score, they found that in patients with PSA $\geq 3 \mathrm{ng} / \mathrm{mL}$, the risk of distant metastases at 5, 10, 15, and 20 years would be greater for the high-risk group with $4 \mathrm{~K} \geq 7.5 \%(2.4 \%, 5.6 \%, 9.9 \%$, and $16.4 \%$ ) than for the low-risk group with $4 \mathrm{~K}<7.5 \%$ $(0 \%, 0.2 \%, 1 \%$, and $1.8 \%)$ [32].

In men with previously negative prostate biopsy but persistently elevated PSA, $4 \mathrm{~K}$ score was found to have superior predictive capacity for high-grade cancers while minimizing unnecessary biopsies [32]. The $4 \mathrm{~K}$ score showed higher discriminatory accuracy than PSA and DRE alone (AUC 0.68 versus $0.58, P<0.001$ ) in detecting $\mathrm{PCa}$ on repeat biopsy. For the prediction of high-grade (Gleason $\geq 7$ ) $\mathrm{PCa}$, the $4 \mathrm{~K}$ score outperformed clinical factors alone (AUC 0.87 versus $0.76 . P=0.003$ ). Using a $4 \mathrm{~K}$ risk threshold of $15 \%, 712$ repeat biopsies could potentially be avoided for every 1000 men, with only 3 of the 53 missed cancer diagnoses having a Gleason score $\geq 7$ [32].

A comparison of $4 \mathrm{~K}$ score and $\mathrm{PHI}$ in a populatedbased cohort study of 531 men with PSA 3 to $15 \mathrm{ng} / \mathrm{mL}$ showed their predictive values to be similar in predicting any PCa (AUC 0.69 for 4K, AUC 0.70 for PHI), as well as high-grade $\mathrm{PCa}(\mathrm{AUC}=71.8$ for $4 \mathrm{~K}, \mathrm{AUC}=71.1 \mathrm{for}$ PHI) [33]. Compared with a base model of age and PSA, 
both $4 \mathrm{~K}$ and PHI had higher AUC $(P<0.001)$. Using high-grade PCa risk thresholds of $10 \%$ for $4 \mathrm{~K}$ and 39 for PHI, 29\% of biopsies could potentially be avoided with the caveat that $10 \%$ of high-grade cancers could be missed [33].

Although there is little evidence to support their use in primary screening, both $\mathrm{PHI}$ and $4 \mathrm{~K}$ scores are mentioned by the European Association of Urology (EAU), American Association of Urology (AUA), and National Comprehensive Cancer Network (NCCN) as potential markers that may be used to risk stratify patients in the early detection of PCa. Their benefits of predicting risk of high-grade $\mathrm{PCa}$ and reducing unnecessary biopsies in men with PSA from 2 to $10 \mathrm{ng} /$ $\mathrm{mL}$ is recognized, as is their superiority over \% $\mathrm{fPSA}$ [35].

\section{Urinary Biomarkers}

\section{Progensa prostate cancer antigen 3 (PCA3)}

PCA3 (Hologic, Marlborough, US) is a non-coding large chain RNA that is highly overexpressed in the majority of malignant prostate tissue compared with benign prostate tissue [36,37]. The Progensa PCA3 gene assay measures PCA3 mRNA concentrations in the first void urine collected after DRE [38]. In 2012, the FDA approved the use of PCA3 to facilitate the decisionmaking process to re-biopsy men with a previous negative biopsy. Multiple studies have evaluated the clinical utility of PCA3 in the early detection of PCa and as a prognostic marker in the active surveillance of patients with low-risk PCa [39-41].

A key European multicenter prospective study by Haese et al. correlated PCA3 scores of 463 men with previous negative prostate biopsies to the repeat biopsy outcomes, with a higher PCA3 score associated with a greater probability of a positive repeat biopsy [39]. Men who had a PCA3 score $\geq 35$ had a $39 \%$ chance of having a positive biopsy compared with $22 \%$ in men with a PCA3 score $<35(P<0.001)$. The mean PCA3 score was significantly higher in men with a positive biopsy than in those with a negative biopsy (63.8 versus 35.5 ; $P<0.001)$. Furthermore, a PCA3 threshold of 35 provided greater diagnostic accuracy than a comparable $\%$ fPSA cutoff of $25 \%$. PCA 3 was found to be independent of tPSA, prostate volume, and patient age [39].

In a retrospective analysis of 13 men, $\mathrm{Wu}$ et al. reported that use of a PCA3 score threshold of 25 yielded sensitivity and specificity of 0.67 and 0.64 , respectively [42]. Although PCA3 was found to be independently associated with PCa (AUC 0.64) in multivariable analyses, the highest diagnostic accuracy was derived from a model comprising PCA3, PSAD, PSA, DRE, and TRUS findings (AUC 0.82) [42].
A prospective validation trial by Wei et al. included 859 PSA-screened patients undergoing initial biopsy and repeat biopsy after prior negative biopsy [41]. The authors demonstrated a positive predictive value (PPV) of $80 \%$ for PCA3 $>60$ at initial biopsy and a negative predictive value (NPV) of $88 \%$ for PCA $3<20$ at repeat biopsy. Their findings supported the use of PCA3 in reducing unnecessary biopsies in men with a prior negative biopsy and concluded that for initial biopsy, a PCA3 $>60$ significantly increases the probability of cancer detection [41].

In another European prospective multicenter study of 516 men, de la Taille et al. observed that the mean PCA3 score was higher in those men with a positive versus negative biopsy (69.6 versus $31.0 ; P<0.001$ ) and higher in men with csPCa. PCA3 scores $>35$ had the highest diagnostic accuracy with sensitivity of $64 \%$ and specificity of $76 \%$. PCA3 score was independent of age, total PSA and prostate volume and outperformed total PSA, PSAD, and \%fPSA [43].

The ability of PCA3 to predict tumor volume and select low-risk patients for active surveillance was prospectively evaluated by Ploussard et al. who reported that PCA3 scores strongly correlated with tumor volume [44]. A PCA3 score $>25$ was associated with a 3 -fold increase in risk of csPCa. On multivariate analysis, a PCA3 score $>25$ was a predictive factor for tumor volume $>0.5 \mathrm{~cm} 3$ (OR 5.4; $P=0.01$ ) and for significant cancer (OR 12.7, $P=0.003$ ), suggesting its use as a tool in selecting better candidates for active surveillance [44]. Nakanishi et al. also found a significant association between PCA3 and tumor volume, with PCA3 being particularly useful in identifying low tumor volume $<$ $0.5 \mathrm{~mL}$ (AUC 0.757) [45].

As PCA3 is the only urinary biomarker with FDA approval, the various guidelines do mention its use to risk stratify patients after a previous negative biopsy and to determine the need for a repeat biopsy. However, it is not recommended as a primary screening tool, and no threshold for the PCA3 score has been defined to guide decision-making.

\section{ExoDx prostate intelliscore (EPI)}

The ExoDx Prostate Intelliscore (Exosome diagnostics, Waltham, US) is a newer, novel urine exosome gene expression assay used to predict the risk of PCa on biopsy. Exosomes are 1 of 2 types of microvesicles found in prostate secretions [46]. Exosomes may be secreted by both normal and malignant tissues, but elevated exosome secretions have been found in malignant biofluids such as the urine of patients with PCa. Exosomes contain a portion of the parent cell cytoplasm containing proteins and RNA that closely resemble the 
cell of origin [47]. Exosomes lack ribosomal RNA but are rich in mRNA, which acts as a unique genetic footprint of specific tumor cells and may give information about the specific tumor genotype that underlies the varying phenotypes that are seen in a heterogenous disease like PCa. Exosomes isolated from post DRE urine contain PCA3 and ERG (erythroblastosis virus E26 oncogene homologues) [47]. The EPI test has the advantage of not requiring a pre-collection DRE, making it non-invasive and more convenient. The test uses an algorithm independent of clinical variables to provide a risk score, with 15.6 being the threshold discriminating between high-grade and low-grade $\mathrm{PCa}$ [48].

McKiernan et al. compared the performance of EPI with biopsy outcomes in men with PSA ranging from 2 to $20 \mathrm{ng} / \mathrm{mL}$, then went on to validate the prognostic score [49]. They found that incorporating EPI into the standard of care (SOC) improved the discrimination of high-grade disease from low-grade or benign disease. Validation in 519 patients showed the superior performance of EPI plus SOC (AUC 0.73) over SOC alone (AUC 0.63) in predicting high-grade disease $(P<0.001)$. Using an EPI cutoff score of 15.6, the authors concluded that $27 \%$ of biopsies could have been avoided, missing $5 \%$ of high-risk cancers [49].

A recent prospective randomized study by Tutrone et al. examined the clinical utility and the impact of EPI score on decision-making in men presenting for initial prostate biopsy with PSA 2 to $10 \mathrm{ng} / \mathrm{mL}$ [48]. A total of 1094 patients and 72 urologists were involved in the study. Sixty-eight percent of urologists were influenced by the EPI score in their decision to recommend or defer a biopsy, the main reason for noncompliance with EPI results being a rising PSA. Eighty-seven percent of patients with a positive EPI score were recommended to proceed to biopsy, leading to the detection of $30 \%$ more high-grade PCa than in the control arm. On the other hand, $63 \%$ of patients with a negative EPI score were recommended to defer prostate biopsy, and the authors estimated that $49 \%$ fewer high-grade cancers were missed because of biopsy deferral compared with SOC [48].

The latest NCCN guidelines do mention EPI as a potential investigative marker, but it is not currently incorporated into mainstream practice.

\section{Mi prostate score (MiPS)}

The MiPS assay was developed at the University of Michigan Rogel Cancer Centre. It is a urine multiplex analysis that combines PSA with $2 \mathrm{PCa}$ specific biomarkers: PCA3 and an RNA marker that is found only when TMPRSS2 and ERG abnormally fuse. ERG is an ET transcription factor that is overexpressed in 57\% of prostate cancers [50]. In more than $90 \%$ of cases that overexpressed ERG, there was found to be fusion with TMPRSS2. TMPRSS2-ERG (T2-ERG) fusion is thought be a strong indicator of $\mathrm{PCa}$.

In a trial of 48 patients undergoing prostate biopsy, Salami et al. found an association between the presence of T2-ERG and PCa (OR 12.02, $P<0.001)$ [51]. Although PCA3 had higher sensitivity (93\%), T2-ERG had the highest specificity (87\%) in predicting PCa. T2-ERG also had better discriminative ability (AUC 0.77) compared with PCA3 (AUC 0.65) and serum PSA (AUC 0.72). Combining all 3 factors into a multivariable algorithm improved the AUC for cancer prediction to 0.88 with specificity of $90 \%$ and sensitivity of $80 \%$, better than any individual marker alone [51]. Laxman et al. showed that compared with PCA3 alone (AUC 0.662), T2-ERG in combination with PCA3 and a multiplex panel of urinary transcripts (AUC 0.758) improved the early detection of $\mathrm{PCa}$ [52].

A validation trial in 1225 patients by Tomlins et al. assessed the ability of the multivariable MiPS model incorporating PSA, PCA3 and T2-ERG in predicting $\mathrm{PCa}$ and high-grade PCa on biopsy [53]. The authors showed that models incorporating T2-ERG had higher AUC than PSA in predicting any (0.693 versus 0.585$)$ and high-grade (0.729 versus 0.651$) \mathrm{PCa}$. The MiPS model incorporating T2-ERG outperformed other models incorporating only PCA3 and PSA in the detection of $\mathrm{PCa}(P<0.001)$ [53].

The MiPS is currently an investigational tool within NCCN guidelines and currently not routinely used in mainstream practice.

\section{Select MDx}

Select MDx (MDx Health, Irvine, US) is a post DRE urine-based gene assay risk score that aims to predict a patient's risk of high-grade PCa. The algorithm measures the mRNA signatures of 2 genes implicated in prostate carcinogenesis-homeobox C6 (HOXC6) and distal-less homeobox 1 (DLX1) - and combines these with clinical factors such as age, family history, previous negative biopsies, and DRE. Leyten et al. initially described a 3-gene urinary panel including HOXC6 and DLX1 that was shown to have additional value over serum PSA and PCA3 in the detection of $\mathrm{PCa}$ and reducing the risk of overtreatment [54].

Van Neste et al. developed a model incorporating HOXC6 and DLX1 on a cohort of 519 patients and subsequently validated the risk score in an independent cohort of 386 patients in two prospective multicenter studies [55]. The authors identified the mRNA signature risk score in combination with PSAD and previous negative biopsies to be the most significant factors with an overall AUC approaching 0.90 (95\% CI 0.85 to 0.95 ). Another model adding DRE as a risk factor 
was also tested with an AUC of 0.86. When compared with the PCPTRC and PCA3, this model could reduce unnecessary biopsies by $53 \%$ with a NPV of $98 \%$ for Gleason $\geq 7$ disease [55].

Correlating Select MDx with mpMRI results, a retrospective observational study of 172 patients reported a positive association between the risk score and the final PI-RADS grade [56]. Median Select MDx scores were higher in patients with a suspicious lesion on mpMRI than in those with a negative mpMRI $(P<0.01)$. Select MDx was also shown to have some value in predicting the mpMRI result with AUC of 0.83 compared with PSA (AUC 0.66) and PCA3 (AUC 0.65).

The cost effectiveness of Select MDx compared with SOC was evaluated by Dijkstra et al., who concluded

\section{References}

1. Cooperberg MR, Carroll PR. Trends in management for patients with localized prostate cancer, 1990-2013. JAMA. 2015;314(1):80-2. doi: 10.1001/jama.2015.6036

2. Wang S-Y, Cowan JE, Cint Cary K, et al. Limited ability of existing nomograms to predict outcomes in men undergoing active surveillance for prostate cancer. BJU Int. 2014; 114(6b):E18-E24. doi.org/10.1111/bju.12554

3. Kretschmer A, Tilki D. Biomarkers in prostate cancer - Current clinical utility and future perspectives. Crit Rev Oncol Hematol. 2017;120:180-193. doi: 10.1016/j.critrevonc.2017.11.007

4. Lonergan PE, Washington SL III, Meng MV, Eapen R. SIU-ICUD Consultation on Molecular Biomarkers in Urological Oncology: the Clinical Applications of Tissue Biomarkers in Prostate Cancer. Soc Int Urol J. 2020;1(1):30-38.

5. Stamey TA, Yang N, Hay AR, et al. Prostate-specific antigen as a serum marker for adenocarcinoma of the prostate. N Engl J Med. 1987;317(15):909-16. doi: 10.1056/NEJM198710083171501

6. Cooner WH, Mosley BR, Rutherford CL Jr, et al. Prostate cancer detection in a clinical urological practice by ultrasonography, digital rectal examination and prostate specific antigen. J Urol. 1990;143(6):1146-52; discussion 1152-4. doi: 10.1016/ s0022-5347(17)40211-4

7. Catalona WJ, Smith DS, Ornstein DK. Prostate cancer detection in men with serum PSA concentrations of 2.6 to $4.0 \mathrm{ng} / \mathrm{mL}$ and benign prostate examination. JAMA. 1997; 277(18):1452.

8. Thompson IM, Pauler DK, Goodman PJ, et al. Prevalence of prostate cancer among men with a prostate-specific antigen level $\leq 4.0 \mathrm{ng}$ per milliliter. N Eng/ J Med. 2004;350(22):2239-2246. doi: 10.1056/ NEJMoa031918

9. Filella X, Foj L, Augé JM, et al. Clinical utility of \%p2PSA and prostate health index in the detection of prostate cancer. Clin Chem Lab Med. 2014;52(9):1347-55. doi: 10.1515/cclm-2014-0027 that the judicious use of Select MDx to reduce the overdiagnosis and overtreatment of men with PCa and PSA $>3 \mathrm{ng} / \mathrm{mL}$ could lead to reduction in costs and gains in quality adjusted life years [57].

\section{Conclusions}

A myriad of serum and urinary biomarkers have emerged with the goal of improving decision-making processes in the early diagnosis of localized PCa. Although a few have gained FDA approval, most are investigational and not used routinely in clinical practice. Biomarkers must add value beyond existing multivariable models to be cost-efficient and of overall benefit. Therefore, at this time, they must be used judiciously in the management of patients with suspected and known PCa.

10. Stenman UH, Leinonen J, Alfthan $H$, et al. A complex between prostate-specific antigen and alpha 1-antichymotrypsin is the major form of prostate-specific antigen in serum of patients with prostatic cancer: assay of the complex improves clinical sensitivity for cancer. Cancer Res. 1991;51(1):222-6.

11. Christensson A, Björk T, Nilsson 0, et al. Serum prostate specific antigen complexed to alpha 1-antichymotrypsin as an indicator of prostate cancer. J Urol. 1993;150(1):100-5. doi: 10.1016/ s0022-5347(17)35408-3

12. Lilja $H$, Christensson A, Dahlén $U$, et al. Prostate-specific antigen in serum occurs predominantly in complex with alpha 1-antichymotrypsin. Clin Chem. 1991;37(9):1618-25.

13. Catalona WJ, Smith DS, Wolfert RL, et al. Evaluation of percentage of free serum prostate-specific antigen to improve specificity of prostate cancer screening. JAMA. 1995;274(15):1214-20.

14. Catalona WJ, Partin AW, Slawinet KM, al. Use of the percentage of free prostate-specific antigen to enhance differentiation of prostate cancer from benign prostatic disease. JAMA. 1998;279(19):1542-7. doi: 10.1001/jama.279.19.1542

15. Stephan $C$, Lein $M$, Jung $K$, et al. The influence of prostate volume on the ratio of free to total prostate specific antigen in serum of patients with prostate carcinoma and benign prostate hyperplasia. Cancer. 1997;79(1):104-9.

16. Benson MC, Whang IS, Pantuck A, et al. Prostate specific antigen density: a means of distinguishing benign prostatic hypertrophy and prostate cancer. J Urol. 1992;147(3 Pt 2):815-6. doi: 10.1016/ s0022-5347(17)37393-7

17. Kundu SD, Roehl KA, Yu X, et al. Prostate specific antigen density correlates with features of prostate cancer aggressiveness. J Urol. 2007;177(2):505-9. doi: 10.1016/j.juro.2006.09.039

18. Teeter AE, Bañez LL, Presti JC Jr, et al. What are the factors associated with short prostate specific antigen doubling time after radical prostatectomy? A report from the SEARCH database group. J Urol. 2008;180(5):1980-1985. doi: 10.1016/j.juro.2008.07.031 
19. Catalona WJ, Partin AW, Sanda MG, et al. A multicenter study of [-2]pro-prostate specific antigen combined with prostate specific antigen and free prostate specific antigen for prostate cancer detection in the 2.0 to $10.0 \mathrm{ng} / \mathrm{ml}$ prostate specific antigen range. J Urol. 2011;185(5):1650-5. doi: 10.1016/j.juro.2010.12.032

20. Stephan C, Vincendeau S, Houlgatte A, et al. Multicenter evaluation of [-2]proprostate-specific antigen and the prostate health index for detecting prostate cancer. 2013;59(1):306-314. doi: 10.1373/ clinchem.2012.195784

21. de la Calle C, Patil D, Wei JT, et al. Multicenter evaluation of the prostate health index to detect aggressive prostate cancer in biopsy naïve men. J Urol. 2015;194(1):65-72. doi: 10.1016/j.juro.2015.01.091

22. Tosoian JJ, Druskin SC, Andreas D, et al. Prostate health Index density improves detection of clinically significant prostate cancer. BJU Int. 2017;120(6):793-798. doi: 10.1111/bju.13762

23. White J, Shenoy BV, Tutrone RF, et al. Clinical utility of the Prostate Health Index (PHI) for biopsy decision management in a large group urology practice setting. Prostate Cancer and Prostatic Dis. 2018;21(1):78-84. doi: 10.1038/s41391-017-0008-7

24. Tosoian JJ, Druskin SC, Andreas D, et al. Use of the Prostate Health Index for detection of prostate cancer: results from a large academic practice. Prostate Cancer Prostatic Dis. 2017;20(2):228233. doi: $10.1038 /$ pcan.2016.72

25. Gnanapragasam VJ, Burling K, George A, et al. The Prostate Health Index adds predictive value to multi-parametric MRI in detecting significant prostate cancers in a repeat biopsy population. Sci Rep. 2016;6(1):35364. doi: 10.1038/srep35364

26. Vickers AJ, Cronin AM, Aus G, et al. A panel of kallikrein markers can reduce unnecessary biopsy for prostate cancer: data from the European Randomized Study of Prostate Cancer Screening in Goteborg, Sweden. BMC Med. 2008;6:19. doi: 10.1186/1741-7015-6-19

27. Benchikh A, Savage C, Cronin AM, et al. A panel of kallikrein markers can predict outcome of prostate biopsy following clinical work-up: an independent validation study from the European Randomized Study of Prostate Cancer screening, France. BMC Cancer. 2010;10(1):635. doi.org/10.1186/1471-2407-10-635

28. Vickers AJ, Cronin AM, Roobol MJ, et al. A Four-Kallikrein Panel Predicts Prostate Cancer in Men with Recent Screening: Data from the European Randomized Study of Screening for Prostate Cancer, Rotterdam. Clin Cancer Res. 2010 Jun 15;16(12):3232-9. doi: 10.1158/1078-0432.CCR-10-0122

29. Vickers AJ, Croninn AM, Aus G, et al. Impact of recent screening on predicting the outcome of prostate cancer biopsy in men with elevated prostate-specific antigen. Cancer. 2010;116(11): 26122620. doi: 10.1002/cncr.25010

30. Vickers A, Cronin AM, Roobol M, et al. Reducing unnecessary biopsy during prostate cancer screening using a four-kallikrein panel: an independent replication. J Clin Oncol. 2010;28(15):2493-2498. doi: 10.1200/JC0.2009.24.1968

31. Parekh DJ, Punnen S, Sjoberg DD, et al. A multi-institutional prospective trial in the USA confirms that the 4kscore accurately identifies men with high-grade prostate cancer. Eur Urol. 2015;68(3):464-470. doi: 10.1016/j.eururo.2014.10.021
32. Stattin P, Vickers A, Sjoberg DD, et al. Improving the specificity of screening for lethal prostate cancer using prostate-specific antigen and a panel of kallikrein markers: a nested case-control study. Eur Urol. 2015; 68(2):207-213. doi: 10.1016/j.eururo.2015.01.009

33. Nordström T, Vickers A, Assel M, et al. Comparison between the four-kallikrein panel and prostate health index for predicting prostate cancer. Eur Urol. 2015;68(1):139-146. doi: 10.1016/j. eururo.2014.08.010

34. Gupta A, Roobol MJ, Savage CJ, et al. A four-kallikrein panel for the prediction of repeat prostate biopsy: data from the European Randomized Study of Prostate Cancer Screening in Rotterdam, Netherlands. Br J Cancer. 2010;103(5):708-714. doi: 10.1038/ sj.bjc. 6605815

35. Mottet N, Bellmunt J, Bolla M, et al. EAU-ESTRO-SIOG guidelines on prostate cancer. Part 1: Screening, diagnosis, and local treatment with curative intent. Eur Urol. 2017;71(4):618-629. doi: 10.1016/j. eururo.2016.08.003

36. Bussemakers MJ, van Bokhoven A, Verhaegh GW, et al. DD3: a new prostate-specific gene, highly overexpressed in prostate cancer. Cancer Res. 1999;59(23):5975-9.

37. Hessels D, Klein Gunnewiek JM, van Oort I, et al. DD3(PCA3)based molecular urine analysis for the diagnosis of prostate cancer. Eur Urol. 2003;44(1):8-15; discussion 15-6. doi: 10.1016/ s0302-2838(03)00201-x

38. Groskopf J, Aubin SMJ, Deras IL, et al. APTIMA PCA3 molecular urine test: development of a method to aid in the diagnosis of prostate cancer. Clin Chem. 2006;52(6):1089-95. doi: 10.1373/ clinchem.2005.063289

39. Haese A, de la Taille A, van Poppel H, et al. Clinical utility of the PCA3 urine assay in European men scheduled for repeat biopsy. Eur Urol. 2008 Nov;54(5):1081-8.

40. Wang F, Ren S, Chen $R$, et al. Development and prospective multicenter evaluation of the long noncoding RNA MALAT-1 as a diagnostic urinary biomarker for prostate cancer. Oncotarget. 2014;5(22):11091-102. doi: 10.18632/oncotarget.2691

41. Wei JT, Feng Z, Partin AW, et al. Can urinary PCA3 supplement PSA in the early detection of prostate cancer? J Clin Oncol. 2014;32(36):4066-4072. doi: 10.1200/JC0.2013.52.8505

42. Wu AK, Reese AC, Cooperberg MR, et al. Utility of PCA3 in patients undergoing repeat biopsy for prostate cancer. Prostate Cancer Prostatic Dis. 2012;15(1):100-5. doi: 10.1038/pcan.2011.52

43. de la Taille A, Irani J, Graefen M, et al. Clinical evaluation of the PCA3 assay in guiding initial biopsy decisions. J Urol. 2011;185(6):2119-2125. doi: 10.1016/j.juro.2011.01.075

44. Ploussard G, Durand X, Xylinas E, et al. Prostate cancer antigen 3 score accurately predicts tumour volume and might help in selecting prostate cancer patients for active surveillance. 2011;59(3):422-429. doi: 10.1016/j.eururo.2010.11.044

45. Nakanishi H, Groskopf J, Fritsche HA, et al. PCA3 molecular urine assay correlates with prostate cancer tumor volume: implication in selecting candidates for active surveillance. J Urol. 2008;179(5):1804-9; discussion 1809-10. doi: 10.1016/j. juro.2008.01.013 
46. Nilsson J, Skog J, Nordstrand A, et al. Prostate cancer-derived urine exosomes: a novel approach to biomarkers for prostate cancer. Br J Cancer. 2009;100(10):1603-1607. doi: 10.1038/sj.bjc.6605058

47. Donovan MJ, Noerholm M, Bentink S, et al. A molecular signature of PCA3 and ERG exosomal RNA from non-DRE urine is predictive of initial prostate biopsy result. Prostate Cancer Prostatic Dis. 2015;18(4):370-375. doi: 10.1038/pcan.2015.40

48. Tutrone R, Donovan MJ, Torkler P, et al. Clinical utility of the exosome based ExoDx Prostate(IntelliScore) EPI test in men presenting for initial Biopsy with a PSA 2-10 ng/mL. Prostate Cancer Prostatic Dis. 2020 May 7. doi: 10.1038/s41391-020-0237-z. Online ahead of print.

49. McKiernan J, Donovan MJ, O'Neill V, et al. A novel urine exosome gene expression assay to predict high-grade prostate cancer at initial biopsy. JAMA Oncol. 2016;2(7):882-9. doi: 10.1001/ jamaoncol.2016.0097

50. Tomlins SA, Rhodes DR, Perner S, et al. Recurrent fusion of TMPRSS2 and ETS transcription factor genes in prostate cancer. Science. 2005;310(5748):644-648. doi: 10.1126/science.1117679

51. Salami SS, Schmidt F, Laxman B, et al. Combining urinary detection of TMPRSS2:ERG and PCA3 with serum PSA to predict diagnosis of prostate cancer. Urol Oncol. 2013;31(5):566-571. doi: 10.1016/j. urolonc.2011.04.001
52. Laxman B, Morris DS, Yu J, et al. A first-generation multiplex biomarker analysis of urine for the early detection of prostate cancer. Cancer Res. 2008;68(3):645-9. doi: 10.1158/0008-5472. CAN-07-3224

53. Tomlins SA, Day JR, Lonigro RJ, et al. Urine TMPRSS2:ERG plus PCA3 for individualized prostate cancer risk assessment. Eur Urol. 2016;70(1):45-53. doi: 10.1016/j.eururo.2015.04.039

54. Leyten GHJM, Hessels D, Smit FP, et al. Identification of a candidate gene panel for the early diagnosis of prostate cancer. Clin Cancer Res. 2015;21(13):3061-3070. doi: 10.1158/1078-0432.CCR-14-3334

55. Van Neste L, Hendriks RJ, Dijkstra S, et al. Detection of highgrade prostate cancer using a urinary molecular biomarkerbased risk score. Eur Urol. 2016;70(5):740-748. doi: 10.1016/j. eururo.2016.04.012

56. Hendriks RJ, van der Leest MMG, Dijkstra S, et al. A urinary biomarker-based risk score correlates with multiparametric MRI for prostate cancer detection. Prostate. 2017;77(14):1401-1407. doi: 10.1002/pros.23401

57. Dijkstra S, Govers TM, Hendriks RJ, et al. Cost-effectiveness of a new urinary biomarker-based risk score compared to standard of care in prostate cancer diagnostics - a decision analytical model. BJU Int. 2017;120(5):659-665. doi: 10.1111/bju.13861 